\title{
Bunch Length Effects in the Beam-Beam Compensation with an Electron Lens
}

\author{
W. Fischer, Y. Luo, C. Montag
}

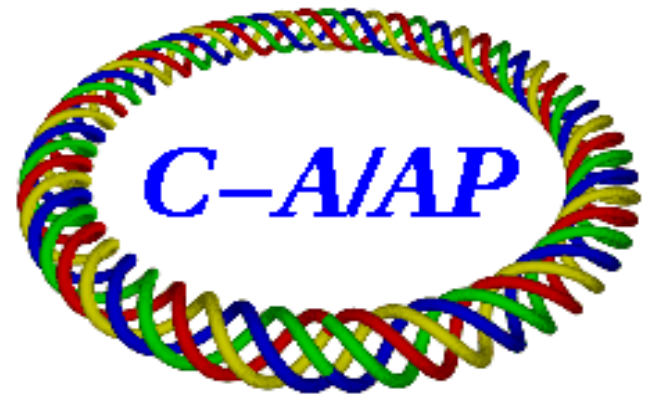

\section{Collider-Accelerator Department Brookhaven National Laboratory Upton, NY 11973}

\footnotetext{
Notice: This document has been authorized by employees of Brookhaven Science Associates, LLC under Contract No. DE-AC02-98CH10886 with the U.S. Department of Energy. The United States Government retains a nonexclusive, paid-up, irrevocable, world-wide license to publish or reproduce the published form of this document, or allow others to do so, for United States Government purposes.
} 


\title{
BUNCH LENGTH EFFECTS IN THE BEAM-BEAM COMPENSATION WITH AN ELECTRON LENS*
}

\author{
W. Fischer ${ }^{\dagger}$, Y. Luo, and C. Montag, \\ Brookhaven National Laboratory, Upton, NY, USA
}

\begin{abstract}
Electron lenses for the head-on beam-beam compensation are under construction at the Relativistic Heavy Ion Collider. The bunch length is of the same order as the $\beta$-function at the interaction point, and a proton passing through another proton bunch experiences a substantial phase shift which modifies the beam-beam interaction. We review the effect of the bunch length in the single pass beam-beam interaction, apply the same analysis to a proton passing through a long electron lens, and study the single pass beam-beam compensation with long bunches. We also discuss the beam-beam compensation of the electron beam in an electron-ion collider ring.
\end{abstract}

\section{INTRODUCTION}

In proton-proton collisions exact beam-beam compensation can be achieved with short bunches and a short electron lens if the following three conditions are met [1]:

1. There are no nonlinearities between the 2 collisions.

2. The phase advance between the p-p and p-e collision is a multiple of $\pi$ in both transverse planes.

3. The proton and the electron beams produce the same amplitude dependent forces by having the same effective charge and transverse profile.

In practice these conditions can only be met approximately. In this article we investigate in which way long bunches affect the beam-beam compensation. We study a system consisting of one beam-beam interaction of a test proton with a long proton bunch followed by a linear transport channel of variable phase advance and a long electron lens.

Our study is motivated by the head-on beam-beam compensation effort for the Brookhaven Relativistic Heavy Ion Collider (RHIC) [1], following the use of electron lenses in the Fermilab Tevatron [2-4]. The beam-beam effects in RHIC are strongest for proton-proton collisions. The rms bunch length is of the same order as the $\beta$-function at the interaction point (IP) (see Tab. 1), and a proton passing through another proton bunch experiences a substantial phase shift which modifies the beam-beam interaction. Fig. 1 shows this for a range of $\beta^{*}$-values. The RHIC electron lenses will operate with a DC current and have a Gaussian transverse profile of constant rms size $\sigma_{e}$. Parameters

\footnotetext{
* Work supported by Brookhaven Science Associates, LLC under Contract No. DE-AC02-98CH10886 with the U.S. Department of Energy.

†Wolfram.Fischer@bnl.gov
}

for both the proton beam and the electron lens of a reference case are shown in Tab. 1.

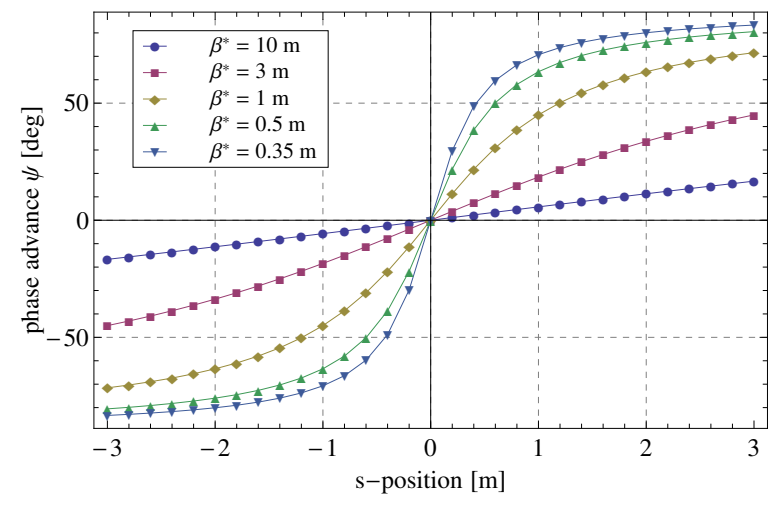

Figure 1: Phase advance near the IP $(s=0)$ for a range of $\beta^{*}$ from $10 \mathrm{~m}$ to $0.35 \mathrm{~m}$ without beam-beam interaction.

We first review the beam-beam interaction of a test particle (proton) with a long round opposing proton bunch. For this we will calculate the change in $\left(r, r^{\prime}\right)$ for various cases, including a time delay $\delta t$ of the test proton, where $r^{\prime}=d r / d s$. The rms bunch length $\sigma_{s}$ and lattice function $\beta^{*}$ of the opposing bunch account for its length, while the time delay $\delta t$ accounts for the length of the other bunch. We then analyze the interaction of a test proton with a long electron lens, and finally study the beam-beam compensation for proton-proton collisions with an electron lens. To make the analysis better tractable we will use the following simplifications:

1. Both proton beams are transversely round at the IP, so that the variable $r$ can stand for either the horizontal $(x)$ or vertical $(y)$ coordinate. Both beams have the same optical functions in the region where the beambeam interaction takes place.

2. There are no magnetic fields other than the ones created by the opposing beam or the electron lens. We disregard the solenoid field in the electron lens.

3. As observation point we chose a symmetry point (IP) where $\alpha^{*}=-\beta^{*} / 2=0$ in both transverse planes. In most cases we will quote $\left(r, r^{\prime}\right)$ in units of the rms beam size $\sigma_{p}(0)$ and the rms angular spread $\sigma_{p}^{\prime}(0)$ at the IP.

We will also discuss the beam-beam compensation in an electron ring of an electron-ion collider [5]. 
Table 1: Reference case for RHIC beam-beam and beamlens interactions with parameters from Ref. [1].

\begin{tabular}{lcc}
\hline \hline quantity & unit & value \\
\hline proton beam parameters & & \\
total energy $E_{p}$ & $\mathrm{GeV}$ & 250 \\
relativistic factor $\beta_{p}$ & $\ldots$ & 0.999993 \\
relativistic factor $\gamma_{p}$ & $\ldots$ & 266.4 \\
bunch intensity $N_{p}$ & $10^{11}$ & 2.0 \\
$\beta_{x, y}^{*}$ at IP6, IP8 $(\mathrm{p}-\mathrm{p})$ & $\mathrm{m}$ & 0.5 \\
$\beta_{x, y}^{*}$ at IP10 $(\mathrm{p}-\mathrm{e})$ & $\mathrm{m}$ & 10.0 \\
lattice tunes $\left(Q_{x}, Q_{y}\right)$ & $\ldots$ & $(.695, .685)$ \\
rms emittance $\epsilon_{n}$, initial & $\mathrm{mm}$ mrad & 2.5 \\
rms beam size at IP6, IP8 $\sigma_{p}^{*}$ & $\mu \mathrm{m}$ & 70 \\
rms beam size at IP10 $\sigma_{p}^{*}$ & $\mu \mathrm{m}$ & 310 \\
rms bunch length $\sigma_{s}$ & $\mathrm{~m}$ & 0.25 \\
rms momentum spread $\delta p / p$ & $10^{-3}$ & 0.30 \\
hourglass factor $F$, initial & $\ldots$ & 0.88 \\
beam-beam parameter $\xi / \mathrm{IP}$ & $\ldots$ & 0.010 \\
number of beam-beam IPs & $\ldots$ & $2+1^{*}$ \\
\hline electron lens parameters & & \\
distance of center from IP & $\mathrm{m}$ & 2.0 \\
effective length $L_{e}$ & $\mathrm{~m}$ & 2.1 \\
kinetic energy $E_{e}$ & $\mathrm{kV}$ & 6.4 \\
relativistic factor $\beta_{e}$ & $\ldots$ & 0.16 \\
relativistic factor $\gamma_{e}$ & $\ldots$ & 1.013 \\
electron line density $n_{e}$ & $10^{11} \mathrm{~m}^{-1}$ & 0.82 \\
electrons in lens $N_{e}$ & $10^{11}$ & 1.7 \\
electrons encountered $N_{e 2}$ & $10^{11}$ & 2.0 \\
current $I_{e}$ & $\mathrm{~A}$ & 0.62 \\
\hline \hline
\end{tabular}

* One head-on collision in IP6 and IP8 each, and a compensating head-on collision in IP10.

\section{SINGLE PASS PROTON INTERACTION WITH LONG BUNCH}

We consider bunches with Gaussian distributions in the longitudinal plane, with rms length $\sigma_{s}$, and both transverse planes with the same rms beam size $\sigma_{x}=\sigma_{y}=\sigma_{p}$. We also assume that the bunches are long compared to the transverse size, $\sigma_{s} \gg \sigma_{p}$. We choose the longitudinal coordinate $s=0$ at the IP, and the time $t=0$ when the opposing proton bunch center passes through $s=0$ from right to left. The test particle shall arrive at $s=0$ at time $\delta t$, so that its time dependent $s$-position is

$$
s=\beta_{p_{1}} c(t-\delta t)
$$

where $c$ is the speed of light and $\beta_{p_{1}}$ the relativistic factor of the test particle. The relativistic factor of the opposing bunch will be denoted by $\beta_{p_{2}}$. We now follow Ref. [6] in our treatment. The radial force for a test particle (proton) in the field of the other bunch is

$$
F_{r}(r)=+\frac{n_{p} e^{2}\left(1+\beta_{p_{1}} \beta_{p_{2}}\right)}{2 \pi \epsilon_{0} r}\left[1-\exp \left(-\frac{r^{2}}{2 \sigma_{p}(s)^{2}}\right)\right]
$$

where $r$ is the radius, $n_{p}$ is the proton line density, $e$ the elementary charge, and $\epsilon_{0}$ the permittivity of vacuum. In the factor $\left(1+\beta_{p_{1}} \beta_{p_{2}}\right)$ the first and second term are from the electric and magnetic fields respectively. The terms are equal if both the test proton and the protons in the opposing bunch are ultra-relativistic. The rms beam size depends on the $s$-position as

$$
\sigma_{p}(s)=\sigma_{p}(0) \sqrt{1+\frac{s^{2}}{\beta^{* 2}}}
$$

where $\beta_{p}^{*}$ is the lattice function at the IP, and the line density $n_{p}$, centered at $s=-\beta_{p_{2}} c t$, is given by

$$
n_{p}(s, t)=\frac{N_{p}}{\sqrt{2 \pi} \sigma_{s}} \exp \left[-\frac{\left(s+\beta_{p_{2}} c t\right)^{2}}{2 \sigma_{s}^{2}}\right] .
$$

$N_{p}$ is the total number of protons in the opposing bunch. The change $d r^{\prime}$ in the radial coordinate $r^{\prime}$ is given by

$$
m_{p} c \beta_{p_{1}} \gamma_{p_{1}} d r^{\prime}=F_{r}(r, s, t) d t
$$

where $m_{p}$ is the proton mass, and $\gamma_{p_{1}}$ is the relativistic factor of the test particle. With Eqs. (1)-(5) the time evolution of $\left(r, r^{\prime}\right)$ is given by the system of non-autonomous ordinary differential equations

$$
\begin{aligned}
\frac{d r}{d t}= & c r^{\prime} \\
\frac{d r^{\prime}}{d t}= & \frac{2 N_{p} r_{p} c\left(1+\beta_{p_{1}} \beta_{p_{2}}\right)}{\sqrt{2 \pi} \beta_{p_{1}} \gamma_{p_{1}} \sigma_{s}} \times \\
& \times \exp \left[-\frac{c^{2}\left(\left(\beta_{p_{1}}+\beta_{p_{2}}\right) t-\beta_{p_{1}} \delta t\right)^{2}}{2 \sigma_{s}^{2}}\right] \times \\
& \times \frac{1}{r}\left[1-\exp \left(-\frac{r^{2}}{2 \sigma_{p}^{2}\left(\beta_{p_{1}} c(t-\delta t)\right)}\right)\right]
\end{aligned}
$$

where we introduced the classical proton radius $r_{p}=$ $e^{2} /\left(4 \pi \epsilon_{0} m_{p} c^{2}\right)$. The function $\sigma_{p}(s)$ is given by Eq. (3). Eqs. (6) can be integrated numerically, for example with the program MATHEMATICA [7]. As an example, Fig. 2 shows the change of $\left(r, r^{\prime}\right)$ of a proton as it passes through a proton bunch with parameters given in Tab. 1 .

We will use the following procedure to compare cases of interest:

1. We consider a test particle with initial coordinates $\left(r_{i}(\delta t), r_{i}^{\prime}(\delta t)\right)$ at the IP $(s=0)$, where $\left(r, r^{\prime}\right)$ are usually given in units of the rms beam size $\sigma_{p}(0)$ and the rms angular spread $\sigma_{p}^{\prime}(0)$ at the IP. $\left(\sigma_{p}(0), \sigma_{p}^{\prime}(0)\right)$ are determined by the rms emittance and the lattice functions at $s=0$ where the lattice is undistorted by the beam-beam lens.

2. We transform the coordinates to a location $s<0$ so that the test particle is outside the field of the opposing bunch.

3. We integrate Eqs. (6) over time $t$ until the test particle is again outside the opposing bunch. 


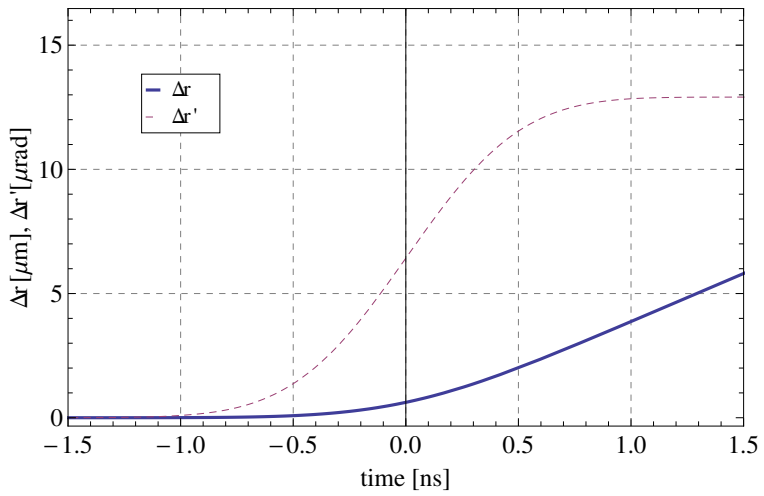

Figure 2: Change of $\left(r, r^{\prime}\right)$ of a proton as a function of time when passing through a long proton bunch at IP6 or IP8. The initial coordinates are $r=1 \sigma_{p}$ and $r^{\prime}=0$. The proton beam parameters are listed in Tab. 1 , and $\delta t=0$.

4. We transform the coordinates back to the IP, using the lattice undisturbed by the beam-beam interaction, resulting in $\left(r_{f}, r_{f}^{\prime}\right)$. The change of coordinates is given by $\left(\Delta r, \Delta r^{\prime}\right)=\left(r_{f}-r_{i}, r_{f}^{\prime}-r_{i}\right)$.

We denote the length of the vector $\left(r, r^{\prime}\right)$ by $R=$ $\sqrt{r^{2}+r^{\prime 2}}$. The result of this integration can then be compared with the case of an infinitely short bunch $\left(\sigma_{s} \rightarrow 0\right)$ and $\delta t=0$, for which we have

$$
\begin{aligned}
\Delta r & =0 \\
\Delta r^{\prime} & =+\frac{2 N_{p} r_{p}\left(1+\beta_{p_{1}} \beta_{p_{2}}\right)}{\beta_{p_{1}} \gamma_{p_{1}}\left(\beta_{p_{1}}+\beta_{p_{2}}\right) r}\left[1-\exp \left(-\frac{r^{2}}{2 \sigma_{p}^{2}(0)}\right)\right] .
\end{aligned}
$$

For this case both $\Delta r$ and $\Delta r^{\prime}$ are independent of $r_{i}^{\prime}$. The change in $r^{\prime}$ as a function of $r$ is shown in Fig. 3 (a).

The numerical integration of Eqs. (6) does not guarantee symplecticity, nor does it account for the energy change of the test particle due to the electric field of the opposing bunch. Our primary interest here is, however, in comparing various situations for a proton passing an opposing bunch or an electron lens only once. A full 6D symplectic treatment of the beam-beam interaction has been devised in Ref. [8] using synchro-beam mapping (SBM). In Ref. [9] the SBM technique was used for the more general case with coupling, through both a tilted beam ellipse of the opposing beam and a solenoid, as well as a crossing angle.

Figure 5 (a) shows the vector $\left(r_{f}, r_{f}^{\prime}\right)$ as a function of $\left(r_{i}, r_{i}^{\prime}\right)$ for an infinitely short bunch, and parts (b) through (d) the deviation from this case for long bunches with parameters in Tab. 1 and $\beta^{*}$-values of $1.0 \mathrm{~m}, 0.5 \mathrm{~m}$, and $0.35 \mathrm{~m}$ respectively. As a general feature long bunches reduce the change in $r^{\prime}$ compared to the short bunch case, and introduce a displacement in $r$. Deviations from the short bunch case are most pronounced for test particles with an initial position $r_{i} \approx 1 \sigma$ and a large initial angle $r_{i}$. For the nominal case of $\beta^{*}=0.5 \mathrm{~m}$ the beam-beam kick is reduced by up to about $1 / 4$ (compare $\Delta R$ in Fig. 5 (c) with $R$

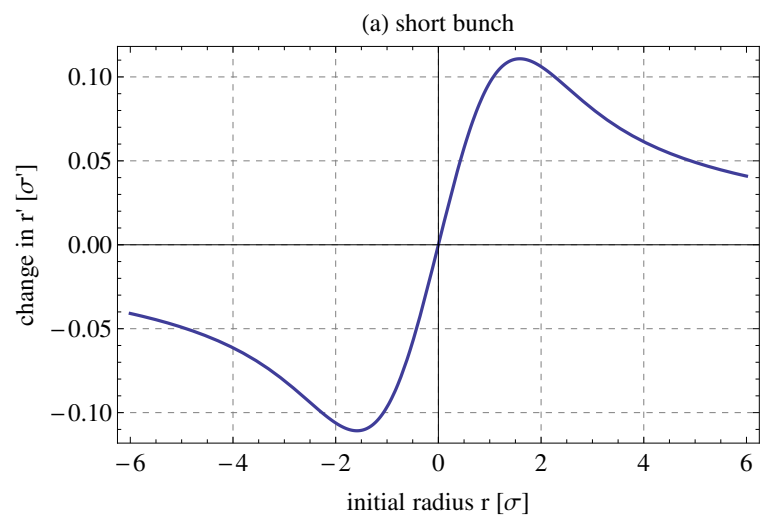

(b) short electron lens at $\mathrm{s}=0$

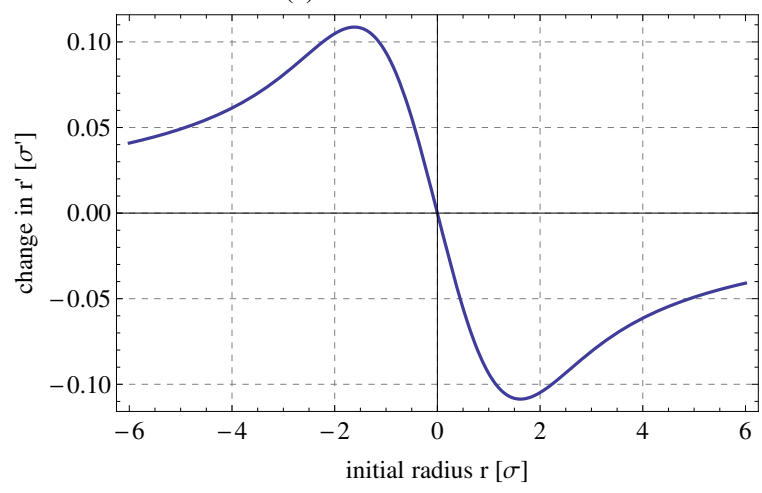

Figure 3: Change in $r^{\prime}$ of a test proton as a function of $r$ due to the beam-beam interaction from an infinitely short bunch in part (a), and from an infinitely short electron lens in part (b). The change in $r^{\prime}$ does not depend on $r^{\prime}$. The kick from a short electron lens has the opposite sign from the kick from a short bunch.

in part (a)). Note that $\beta^{*}$-values from $10 \mathrm{~m}$ to $0.7 \mathrm{~m}$ were used in RHIC, and $\beta^{*}$-values as low as $0.35 \mathrm{~m}$ are under consideration for future upgrades.

Figure 6 shows the effect of the test particle delay $\delta t$, for values ranging from $-3 \sigma_{t}$ to $+3 \sigma_{t}$. For the largest $\delta t$ values and compared to the short bunch case, the beambeam kicks are almost reversed for initial position $r_{i} \approx 1 \sigma$ and large initial angles $r_{i}$.

\section{SINGLE PASS PROTON INTERACTION WITH LONG ELECTRON LENSES}

Different from the opposing beam case treated above, the electrons in a lens are non-relativistic, the electron beam in the lens has a constant transverse rms size $\sigma_{e}$, and the line density $n_{e}$ is also constant for DC lenses like in RHIC. To accomodate two lenses in a common beam pipe section, the RHIC electron lenses are placed close to but not at the IP 
(see Tab. 1). With this the equivalent of Eqs. (6) is

$$
\begin{aligned}
\frac{d r}{d t} & =c r^{\prime} \\
\frac{d r^{\prime}}{d t} & =-\frac{2 n_{e} r_{p} c\left(1+\beta_{p_{1}} \beta_{e}\right)}{\beta_{p_{1}} \gamma_{p_{1}}} \times \frac{1}{r}\left[1-\exp \left(-\frac{r^{2}}{2 \sigma_{e}^{2}}\right)\right]
\end{aligned}
$$

which is independent of the test particle delay $\delta t$ and the proton lattice functions (the initial conditions for the test particle are, however, dependent on the proton lattice functions). In the factor $\left(1+\beta_{p_{1}} \beta_{e}\right)$ the first and second term are again from the electric and magnetic fields respectively. Since the electrons are not highly relativistic (Tab. 1), the electrical forces dominate. The electron line density $n_{e}$ is constant over the length of the electron lens. We disregard the effect of the solenoid field of the electron lens on the test particle. As for the beam-beam interaction case we use the coordinates $\left(r, r^{\prime}\right)$ transformed to the IP for reference, both before and after the test particle interaction with the electron lens. The integration of Eqs. (8) extends over the length of the electron lens $L_{e}$, i.e. the time $L_{e} /\left(\beta_{p} 1 c\right)$. As an example, Fig. 4 shows the change of $\left(r, r^{\prime}\right)$ of a proton as it passes through an electron lens with parameters given in Tab. 1.

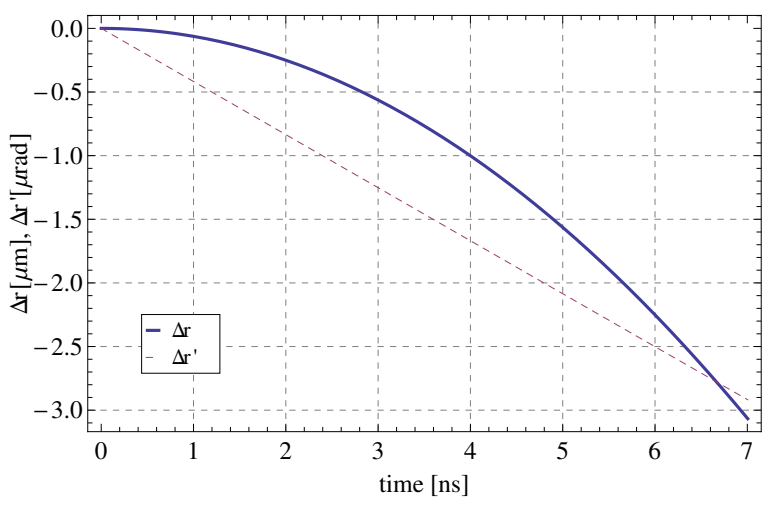

Figure 4: Change of $\left(r, r^{\prime}\right)$ of a proton as a function of time when passing through a long electron lens near IP10. The initial coordinates are $r=1 \sigma_{p}$ and $r^{\prime}=0$. The electron lens parameters are listed in Tab. 1, and the result is independent of $\delta$.

The electron lens is characterized by the rms beam size of the electron beam $\sigma_{e}$, and its integrated strength $N_{e 1}=$ $\left(n_{e} L_{e}\right)$, which is also the number of electrons in the lens. The strength can also be expressed through the number of electrons that the test particle encounters during passage $N_{e 2}=N_{e 1}\left(1+\beta_{e} / \beta_{p 1}\right)$, or the electron current $I_{e}=e n_{e} \beta_{e} c$. A test particle encounters the same number of electrons and protons if $N_{e 2}=N_{p}$, or

$$
I_{e}=\left(\frac{N_{p}}{L_{e}}\right) \frac{e \beta_{e} c}{1+\beta_{e} / \beta_{p 1}} .
$$

As for the beam-beam case we compare the cases of a long electron lens to the cases of a zero length electron lens. The zero length case is shown in Fig. 3 (b). The change in the angle $r^{\prime}$ only depends on the initial radius $r_{i}$. Note that even for a zero length lens, the position will change if the lens is not at the IP, but the final coordinates are transformed back to the IP, as we do.

Figure 7 part (a) shows the vector $\left(r_{f}, r_{f}^{\prime}\right)$ as a function of $\left(r_{i}, r_{i}^{\prime}\right)$ for an infinitely short lens, and parts (b) through (d) the deviation from this case for long lenses with parameters in Tab. 1 and $\beta^{*}$-values of $20 \mathrm{~m}, 10 \mathrm{~m}$, and $5 \mathrm{~m}$ respectively. For the electron lens the result is independent of $\delta t$. As for the beam-beam interaction, the change in $r^{\prime}$ is reduced with a long electron lens, and an additional displacement in $r$ is observed. In the parameter range tested, deviations from the short lens case are not larger than a few percent. The largest deviations are again observed for test particles with $r_{i} \approx 1 \sigma$ and a large initial angle $r_{i}^{\prime}$.

A large $\beta^{*}$ at the location of the electron lens makes alignment of the two beams easier. The planned $\beta^{*}$ at the IP near the electron lens is given in Tab. 1, and is in the middle of the scanned range.

\section{SINGLE PASS HEAD-ON BEAM-BEAM COMPENSATION}

We now consider a beam-beam interaction with a long bunch at IP8 followed by a linear transport channel and an interaction with a long electron lens near IP10. We use the parameters in Tab. 1 and scan four parameters: the phase advance between IP8 and the center of the electron lens, the electron beam size, the electron current, and the time delay $\delta t$. The phase advance between IP8 and the center of the electron lens is denoted by

$$
\psi=\pi+\Delta \psi
$$

The electron beam size is given by

$$
\sigma_{e}=\sigma_{e 0}+\Delta \sigma_{e} \text { with } \sigma_{e 0}=\sqrt{\frac{\epsilon_{n} \beta_{c l}}{\beta_{p 2} \gamma_{p 2}}} .
$$

$\sigma_{e 0}$ is the electron beam size that matches the proton beam size and $\beta_{c l}$ is the lattice function of the test particle lattice in the center of the electron lens. The electron current is given by

$$
I_{e}=I_{e 0}+\Delta I_{e} \text { with } I_{e 0}=\left(\frac{N_{p}}{L_{e}}\right) \frac{e \beta_{e} c}{1+\beta_{e} / \beta_{p 1}}
$$

$I_{e 0}$ is the current that matches the proton bunch intensity $N_{P}$ of the opposing beam. Note that for a short bunch and electron lens, and $\Delta \psi=0, \Delta \sigma_{e}=0, \Delta I_{e}=0$ the head-on beam-beam compensation is exact.

Figure 8 shows in part (a) the final $\left(r_{f}, r_{f}^{\prime}\right)$ of a proton at IP10 after interaction with a long bunch, a linear transport channel of varying phase advance, and a long electron lens. All other parameters are given by Tab. 1 . The initial $\left(r_{i}, r_{i}^{\prime}\right)$ are taken at IP8. Parts (b) through (f) show the $\left(r_{f}, r_{f}^{\prime}\right)$ for phase advance errors $\Delta \psi$ from -30 deg to $+30 \mathrm{deg}$. As 
expected from Fig. 5 the compensation is least effective for test particles with $r_{i} \approx 1 \sigma$ and a large initial angle $r_{i}^{\prime}$. For these and $\Delta \psi=0$ about $1 / 4$ of the beam-beam kick remains uncompensated. A realistic phase advance error is about 10 deg.

Figure 9 shows a scan of the electron beam size $\sigma_{e}$ from $\Delta \sigma_{e} / \sigma_{e 0}=+100 \%$ to $-20 \%$, with a phase advance error of $\Delta \psi=-10 \mathrm{deg}$. A large electron beam size makes the HOBBC ineffective (compare Fig. 8 (a) with Fig. 9 (a)). During commissioning of the electron lens, one can therefore start with a large electron beam that is then tightened. We expect that electron and proton beam sizes can be matched within about $20 \%$. At this value particles with $r_{i} \approx 1 \sigma$ and a large initial angle $r_{i}^{\prime}$ receive only about half the compensation. We use $\Delta \sigma_{e} / \sigma_{e 0}=+20 \%$ for the next scans.

Figure 10 shows a scan of the electron beam current $I_{e}$ from $\Delta I_{e} / I_{e 0}=+50 \%$ to $-50 \%$. A small electron beam current turns off the HOBBC (compare Fig. 8 (a) with Fig. 10 (f)). During commissioning of the electron lens, one can therefore start with a small electron beam current that is then increased. We expect that electron and proton beam currents can be matched within about $5 \%$, where the error stems primarily from bunch-by-bunch variations in the proton intensity. With $\Delta I_{e} / I_{e 0}=5 \%, r_{i} \approx 1 \sigma$ and a large initial angle $r_{i}^{\prime}$ receive only about half the compensation. We use $\Delta I_{e} / I_{e 0}=-5 \%$ for the next scan.

Finally Fig. 11 shows the HOBBC for test particles with $\delta t \neq 0$. Parts (a), (c), and (e) show $\left(r_{f}, r_{f}^{\prime}\right)$ at IP10 without $\mathrm{HOBBC}$ for $\delta t=-3 \sigma_{t},-1 \sigma_{t}$, and $+3 \sigma_{t}$ respectively. Parts (b), (d), and (f) show the $\left(r_{f}, r_{f}^{\prime}\right)$ with $\mathrm{HOBBC}$ with $\Delta \psi=-10 \mathrm{deg}, \Delta \sigma_{e} / \sigma_{e 0}=+20 \%$, and $\Delta I_{e} / I_{e 0}=-5 \%$. Here too we find that the compensation is ineffective for particles with $r_{i} \approx 1 \sigma$ and a large initial angle $r_{i}^{\prime}$.

\section{ELECTRON-ION COLLIDER BEAM-BEAM COMPENSATION}

The beam-beam parameter experienced by the electron beam in an electron-ion collider is typically an order of magnitude higher than in an ion-ion collider. Together with the long ion bunch length, this results in the electrons performing a sizeable fraction of a betatron oscillation period within the proton bunch. This can be parameterized by the disruption parameter $[12,13]$

$$
D=\frac{2 r_{e} N_{p} \sigma_{s}}{\gamma_{e} \sigma_{x} \sigma_{y}}
$$

where $r_{e}$ is the classical electron radius and $\gamma_{e}$ the electron relativistic factor. The betatron phase advance across a beam-beam interaction can then be expressed in terms of the disruption parameter as [12]

$$
\Delta \psi=2 \pi \cdot 2.52 \sqrt{D}
$$

Using the parameters of the eRHIC ring-ring design (Tab. 2), we can compute the betatron phase advance within
Table 2: Parameters of the ring-ring electron-ion collider eRHIC [14].

\begin{tabular}{lcc}
\hline \hline quantity & unit & value \\
\hline electron energy $E_{e}$ & $\mathrm{GeV}$ & 10 \\
relativistic factor $\gamma_{e}$ & $\ldots$ & 19570 \\
electron emittance $\left(\epsilon_{x}, \epsilon_{y}\right)$ & $\mathrm{nm}$ & $(53,9.5)$ \\
proton bunch intensity $N_{p}$ & $10^{11}$ & 2.0 \\
proton bunch length $\sigma_{s}$ & $\mathrm{~m}$ & 0.2 \\
rms beam size at IP $\left(\sigma_{x}, \sigma_{y}\right)$ & $\mu \mathrm{m}$ & $(101,50.5)$ \\
\hline \hline
\end{tabular}

the long ion bunch as $\Delta \psi=2 \pi \cdot 0.6=216$ degrees.

In the limit of infinitely short ion bunches, the phase advance between IP and electron lens was found to have to be accurate within 2 degrees [5], which is small compared to the betatron phase advance $\Delta \psi$ inside the ion bunch.

The elliptical cross section of the beams at the IP, $\sigma_{x}>$ $\sigma_{y}$, complicates the analysis presented for a $\mathrm{p}$-p collider. On the other hand, the case with $\delta t=0$ is sufficient due to the short electron bunch length of only . Simulation studies are currently being performed to investigate the feasibility of head-on beam-beam compensation in the case of long ion bunches. The results of this study will be presented in a separate paper.

\section{SUMMARY}

We have investigated the bunch length effect in the headon beam-beam compensation. The head-on beam-beam interaction with long bunches is modified compared to the short bunch case since the betatron phase changes significantly during the beam-beam interaction. The deviation from the short bunch case is most pronounced for particles with initially large $r^{\prime}$ and $r \approx 1 \mathrm{rms}$ beam size. For these particles the beam-beam effect is reduced; for the parameters in this study (Tab. 1) by about $10 \%$.

The beam-beam interaction with long bunches is further modified for test particles that arrive with a time delay. For the parmeter set under study, particles initially large $r^{\prime}$ and $r \approx 1 \mathrm{rms}$ beam size the changes in $\left(r, r^{\prime}\right)$ change sign compared to the short bunch case.

The beam-beam effect in a long electron lens is also modified compared to a short electron lens. For the parameters in this study (Tab. 1) this effect is by an order of magnitude smaller than in the head-on beam-beam interaction. This is due to the fact that the $\beta$-function at the electron lens is as large as possible to minimize the requirements for the alignment between the electron and proton beams.

Using a single beam-beam interaction per turn and a single electron lens, and expected with the previous results, head-on beam-beam compensation for particles with initially large $r^{\prime}$ and $r \approx 1 \mathrm{rms}$ beam size is not effective in a single pass, in particular for particles with large time delays. Compensation should still be effective when averaged over many turns.

We used the single pass head-on compensation calcu- 
lations to establish goals for the betatron phase error between the beam-beam interaction and the electron lens $(\Delta \psi \leq 10 \mathrm{deg})$, the beam size error of the electron beam $\left(\Delta \sigma_{e} / \sigma_{e 0} \leq 20 \%\right)$ and the current error in the electron beam $\left(\Delta I_{e} / I_{e 0} \leq 5 \%\right)$. The commissioning would start with a large electron beam of small current, and then reduce the size and increase the current.

A full investigation of the effects of long bunches requires long-term tracking of many particles, beyond the scope of this paper. We also note that the modification of resonance driving terms due to the bunch length has been investigated in Ref. [11], where a reduction in the resonance driving term with longer bunches was found.

\section{ACKNOWLEDGMENTS}

The authors are thankful for discussions of long bunch beam-beam problems to H.-J. Kim, J.-P. Koutchouk, J. Qiang, T. Sen, G. Sterbini, A. Valishev, and F. Zimmermann.

\section{REFERENCES}

[1] W. Fischer, Y. Luo, and A. Pikin (eds.) et al. "RHIC electron lenses design report", in preparation.

[2] V. Shiltsev, Y. Alexahin, K. Bishofberger, V. Kamerdzhiev, G. Kuznetsov, and X.-L. Zhang, Phys. Rev. Lett. 99, 244801 (2007).

[3] V. Shiltsev et al., Phys. Rev. ST Accel. Beams 11, 103501 (2008).

[4] X.-L. Zhang, K. Bishofberger, V. Kamerdzhiev, V. Lebedev, V. Shiltsev, R. Thurman-Keup, and A. Tollestrup, Phys. Rev. ST Accel. Beams 11, 051002 (2008).

[5] C. Montag and W. Fischer, Phys. Rev. ST Accel. Beams 12, 084001 (2009).

[6] E. Keil, CERN 95-06, pp. 539-555 (1995).

[7] www.wolfram.com/mathematica

[8] K. Hirata, H. Moshammer, and F. Ruggiero, Part. Accel. 40, pp. 205-228 (1993); SLAC-PUB-10055, KEK-PREPRINT92-117 (1992).

[9] L.H.A. Leunissen, G. Ripken, and F. Schmidt, CERN LHC Project Report 369 (2000).

[10] G.H. Hoffstaetter and F. Willeke, EPAC'02, Paris, France, pp. $401-403$ (2002).

[11] S. Krishnagopal and R. Siemann, Phys. Rev. D, 41, pp. 2312-2316 (1990).

[12] R. J. Hollebeek, Nucl. Instr. Meth A 184, 333 (1981)

[13] S. A. Heifets, G. Krafft and M. Fripp, Nucl. Instr. Meth A 295, 286 - 290 (1990)

[14] M. Farkhondeh, V. Ptitsyn (eds.), C-A/AP/142 (2004). 

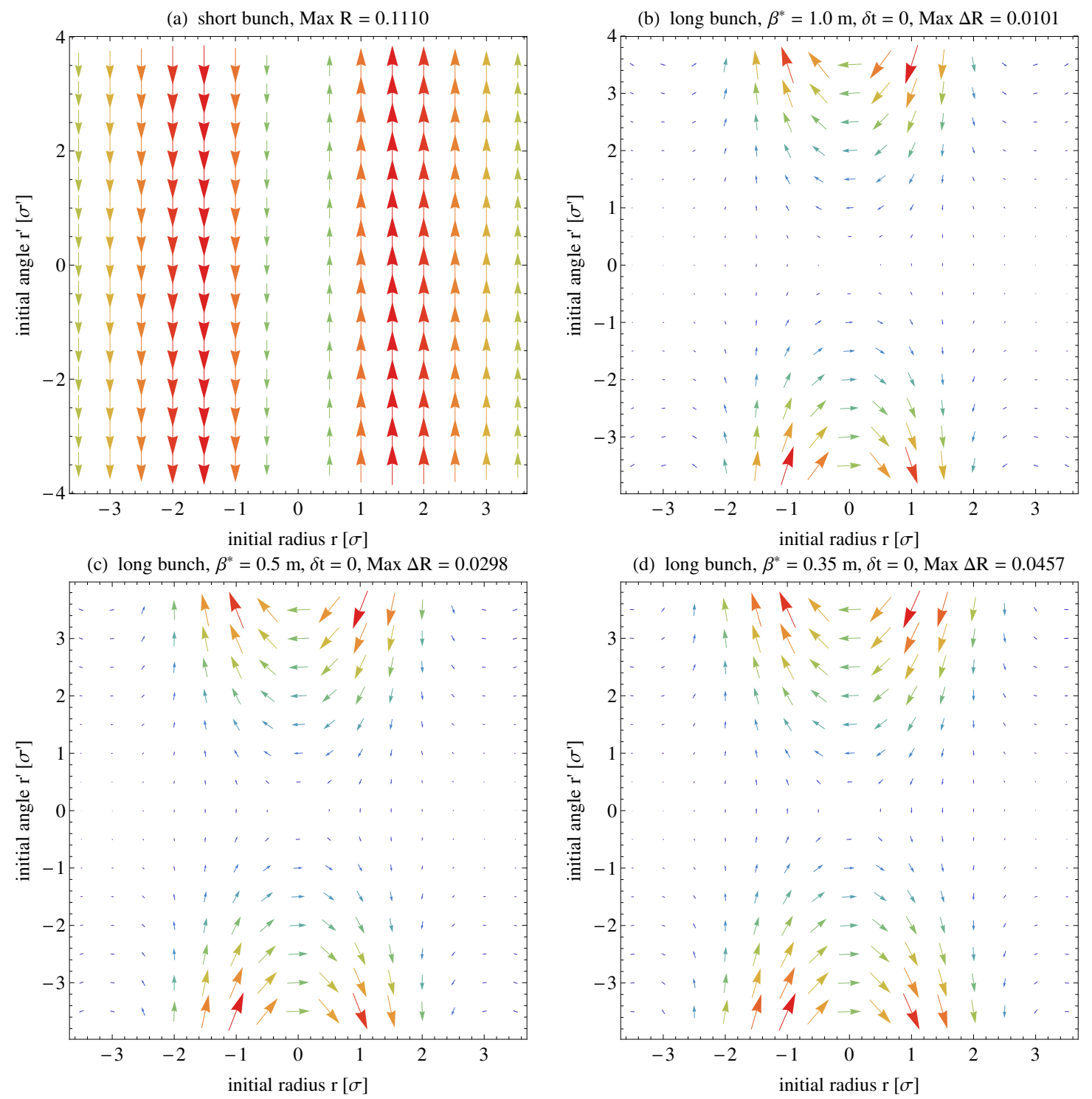

Figure 5: Change of $\left(r, r^{\prime}\right)$ of a proton with $\delta t=0$ through the interaction with a long proton bunch. Part (a) shows the case of an infinitely short bunch, and parts (b) through (d) the deviation from the short bunch case (a). For the long bunches parameters from Tab. 1 are used and $\beta^{*}$ is varied from $1.0 \mathrm{~m}$ to $0.35 \mathrm{~m} . R$ and $\Delta R$ are the lengths of the vectors shown in parts (a) and (b)-(d) respectively. Take note of the $R$ and $\Delta R$ values. 
(a) long bunch, $\beta^{*}=0.5 \mathrm{~m}, \delta \mathrm{t}=-3 \sigma_{t}$, Max $\Delta \mathrm{R}=0.1810$

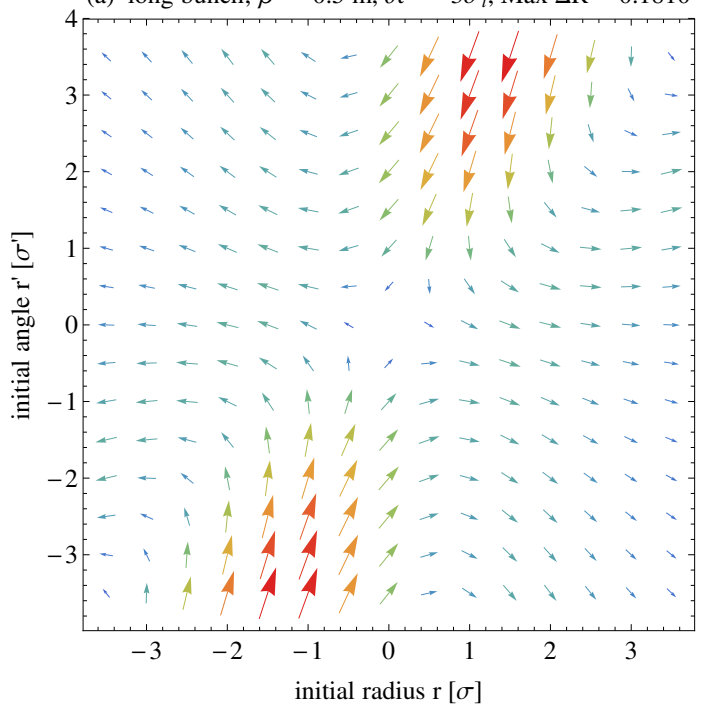

(b) long bunch, $\beta^{*}=0.5 \mathrm{~m}, \delta \mathrm{t}=-1 \sigma_{t}$, Max $\Delta \mathrm{R}=0.0971$

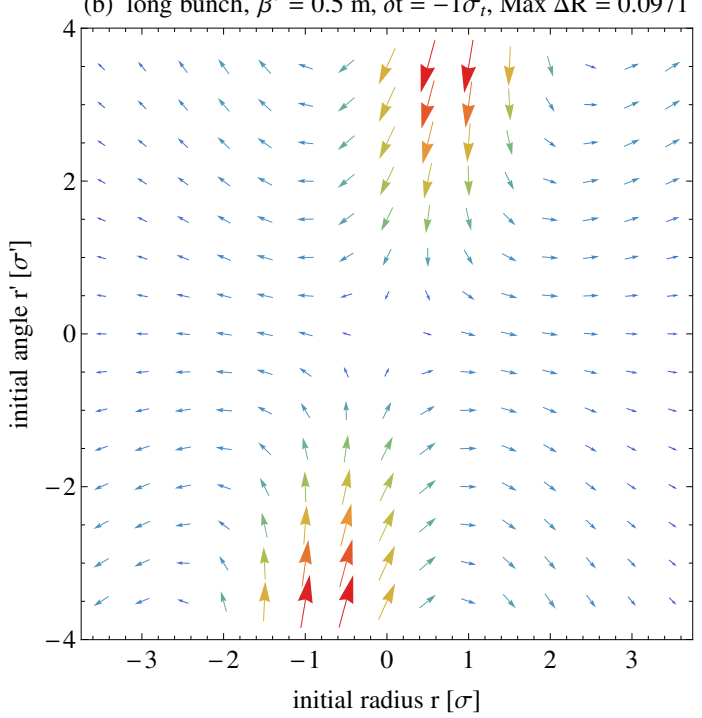

(c) long bunch, $\beta^{*}=0.5 \mathrm{~m}, \delta \mathrm{t}=0 \sigma_{t}$, Max $\Delta \mathrm{R}=0.0298$
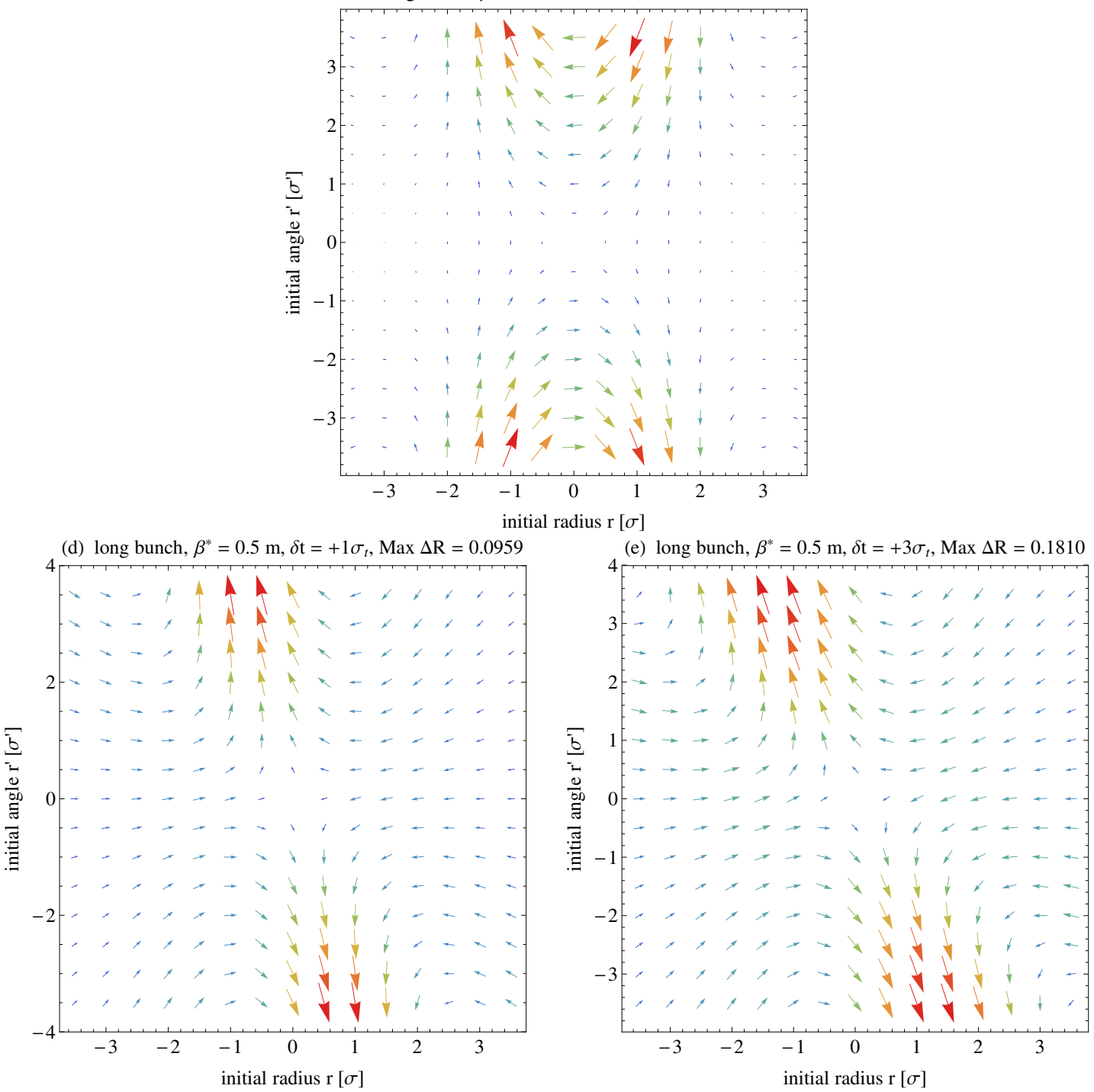

Figure 6: Effect of the particle delay $\delta t$ on the change of $\left(r, r^{\prime}\right)$ of a proton passing through a long proton bunch. Parts (a) through (e) show the deviation to the short bunch case in Fig. 5 (a) for $\delta t$ from $-3 \sigma_{t}$ to $+3 \sigma_{t}$. Take note of the $\Delta R$ values. 

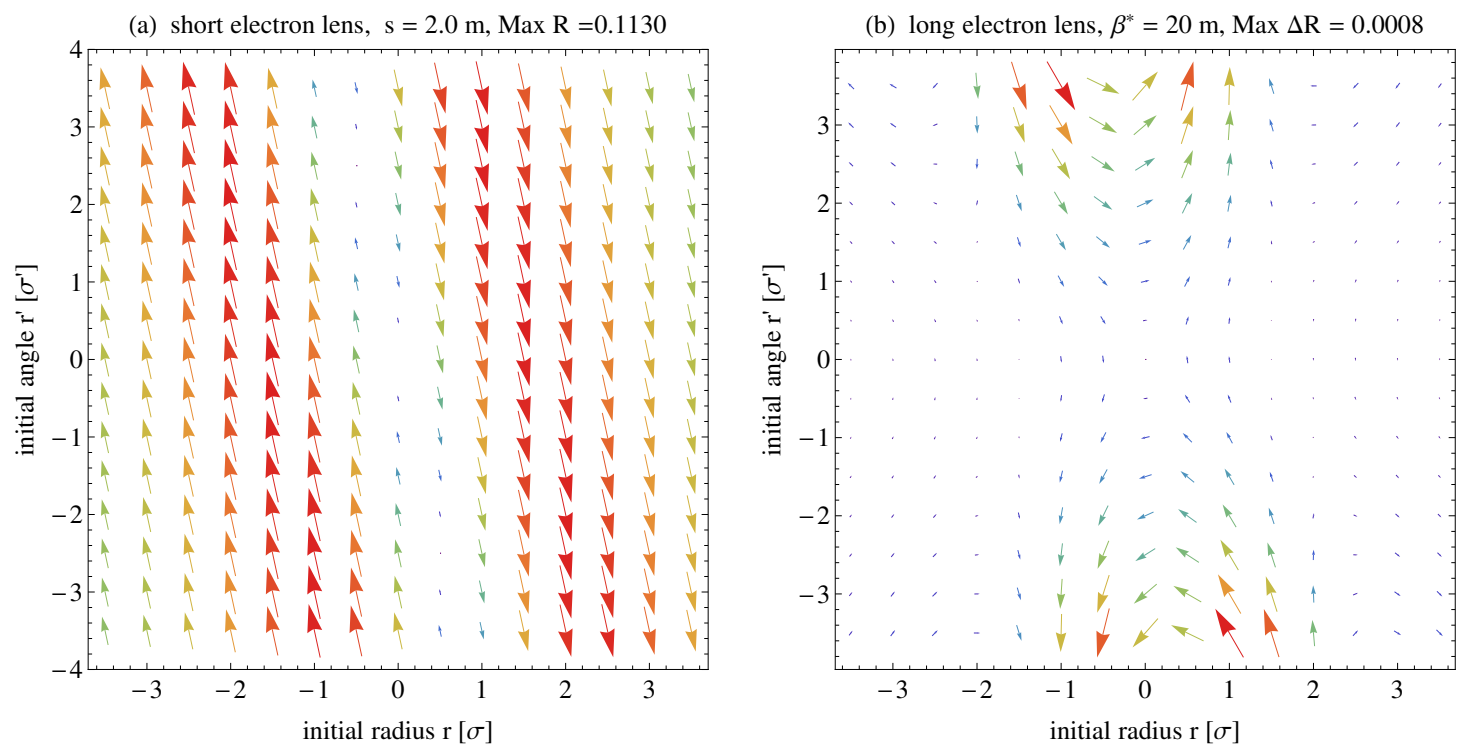

(c) long electron lens, $\beta^{*}=10 \mathrm{~m}$, Max $\Delta \mathrm{R}=0.0028$
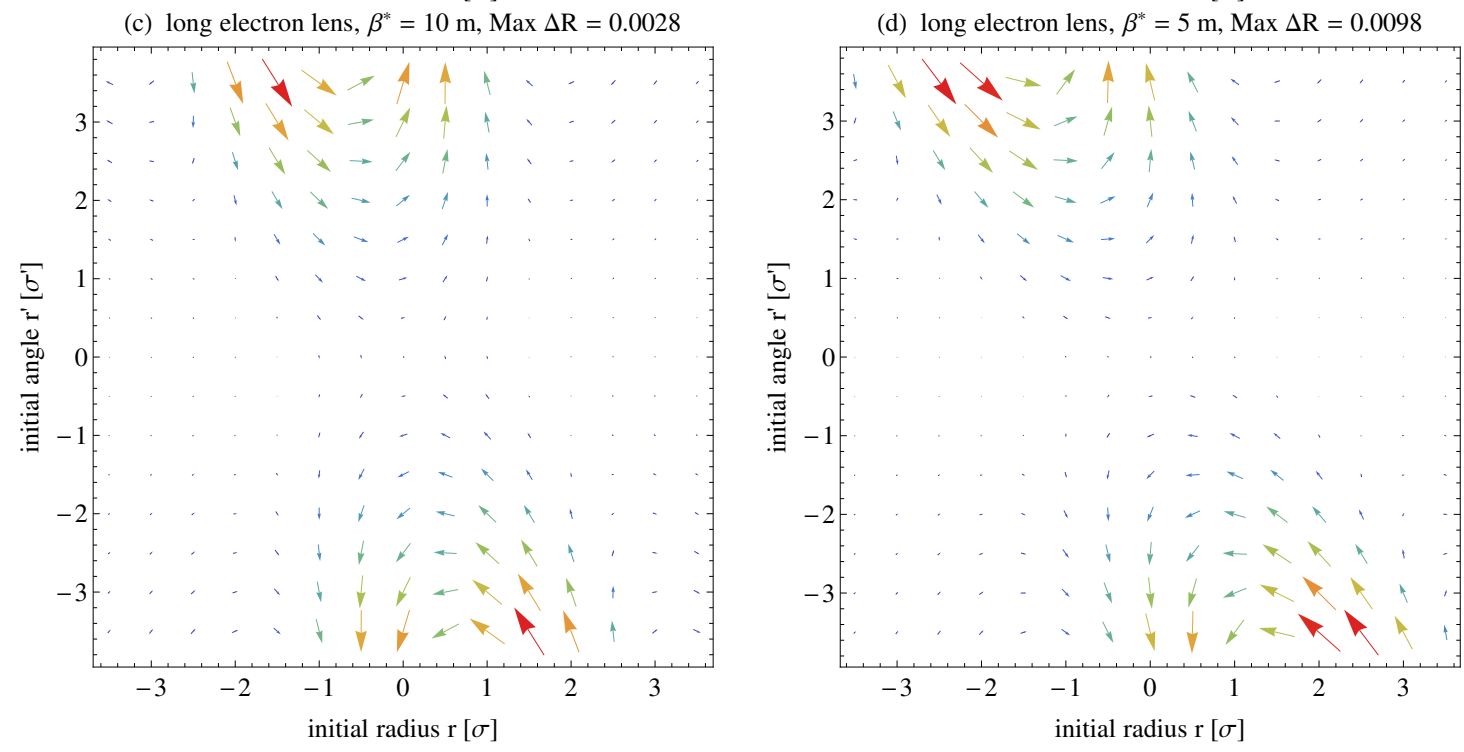

Figure 7: Change of $\left(r, r^{\prime}\right)$ of a proton through the interaction with a long electron lens at $s=1.55 \mathrm{~m}$ from the IP. Part (a) shows the the case of an infinitely short electron lens, and parts (b) through (d) the deviation from the short electron lens case (a). For the long electron lens parameters from Tab. 1 are used and $\beta^{*}$ is varied from $20.0 \mathrm{~m}$ to $0.5 \mathrm{~m}$. $R$ and $\Delta R$ are the lengths of the vectors shown in part (a) and (b)-(d) respectively. Take note of the $R$ and $\Delta R$ values. 


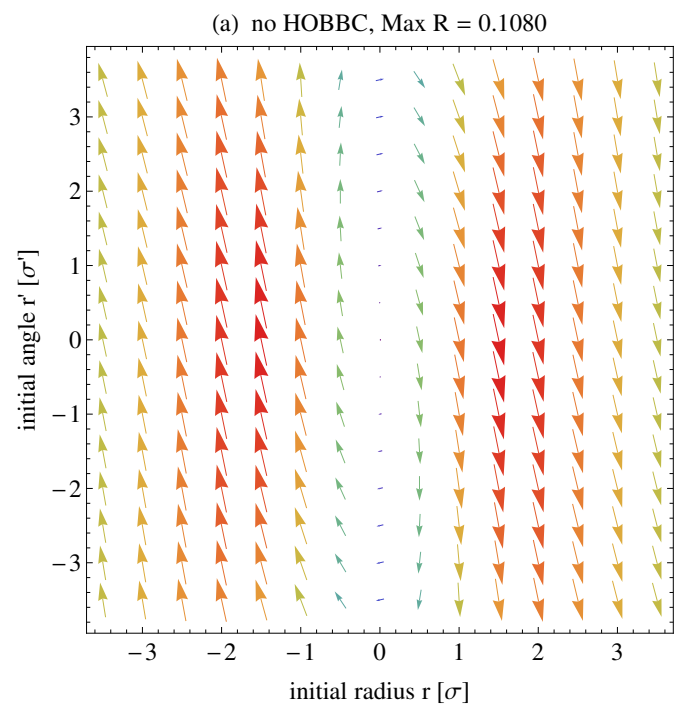

(c) HOBBC, $\Delta \psi=-20 \mathrm{deg}$, Max $\mathrm{R}=0.1120$

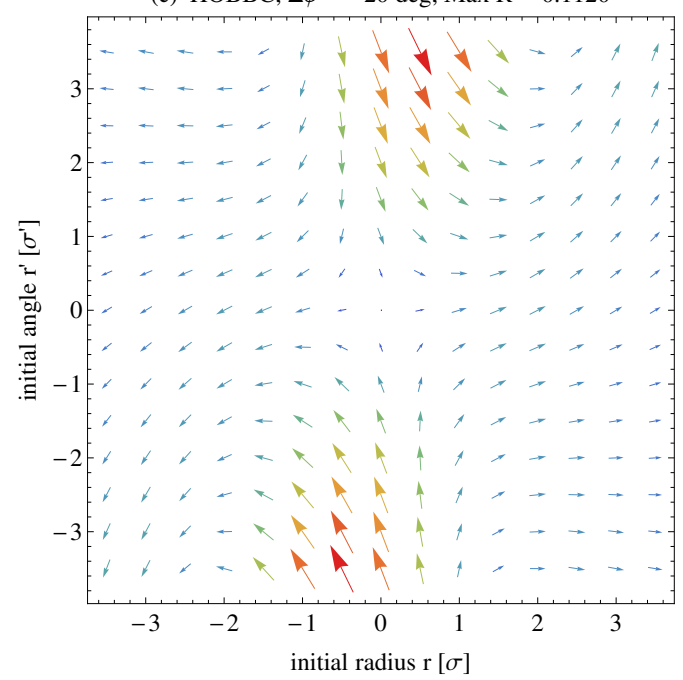

(e) $\mathrm{HOBBC}, \Delta \psi=0$ deg, Max $\mathrm{R}=0.0268$

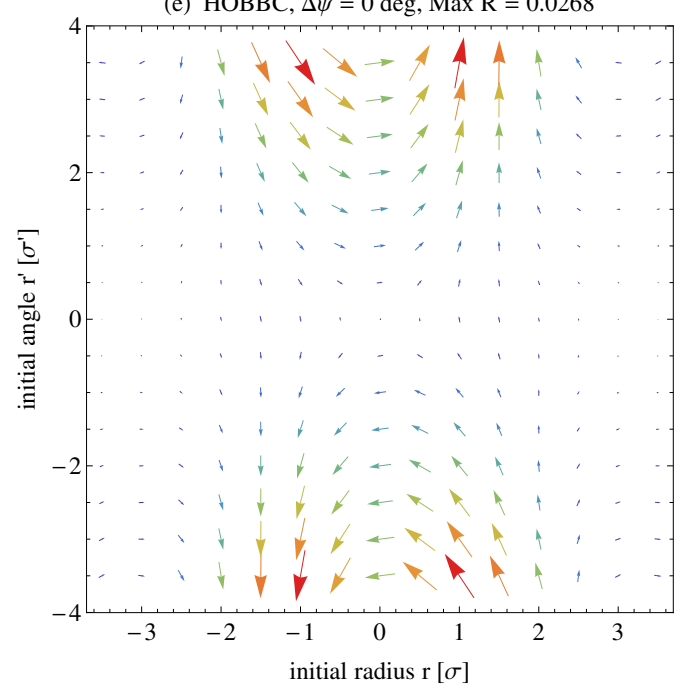

(b) $\mathrm{HOBBC}, \Delta \psi=-30 \mathrm{deg}$, Max $\mathrm{R}=0.1500$

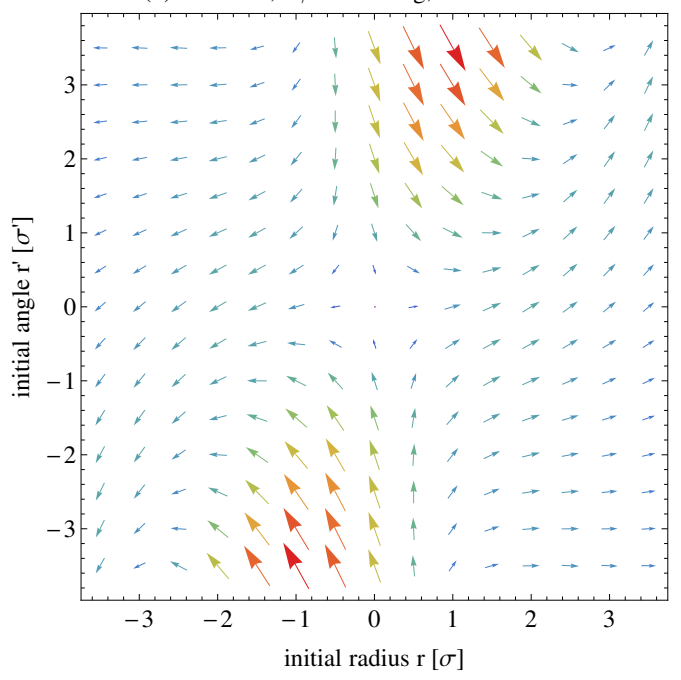

(d) HOBBC, $\Delta \psi=-10 \mathrm{deg}$, Max $\mathrm{R}=0.0656$

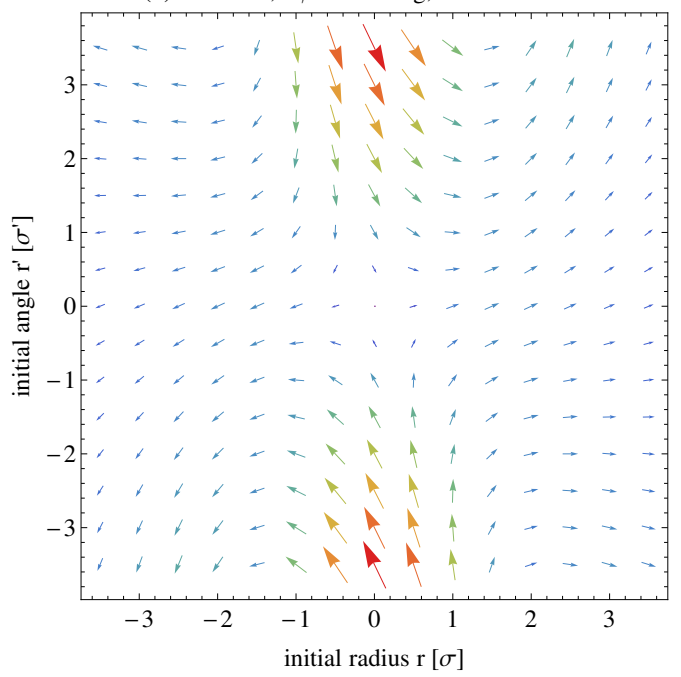

(f) $\mathrm{HOBBC}, \Delta \psi=+30 \mathrm{deg}$, Max $\mathrm{R}=0.1470$

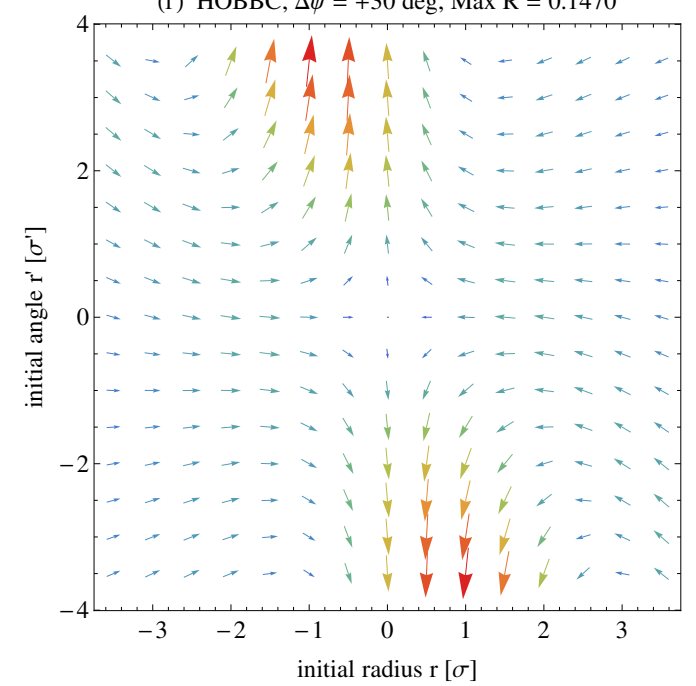

Figure 8: $\left(r_{f}, r_{f}^{\prime}\right)$ of a proton at IP10 after interaction with a long bunch, a linear transport channel of varying phase advance, and a long electron lens. Part (a) shows $\left(r_{f}, r_{f}^{\prime}\right)$ at IP10 as a function of the initial $\left(r_{i}, r_{i}^{\prime}\right)$ at IP8 without headon beam-beam compensation (HOBBC). Parts (b) through (f) show $\left(r_{f}, r_{f}^{\prime}\right)$ with HOBBC and varying phase advance between the beam-beam interaction and the center of the electron lens, given by $\psi=\pi+\Delta \psi$. Proton beam and electron lens parameters are given in Tab. 1 and $\Delta \psi$ is varied between $-30 \mathrm{deg}$ and $+30 \mathrm{deg}$. Take note of the $R$ values, $R \equiv 0$ for perfect compensation. 
(a) HOBBC, $\Delta \sigma_{e} / \sigma_{\mathrm{e} 0}=+100 \%$, Max $\mathrm{R}=0.0712$

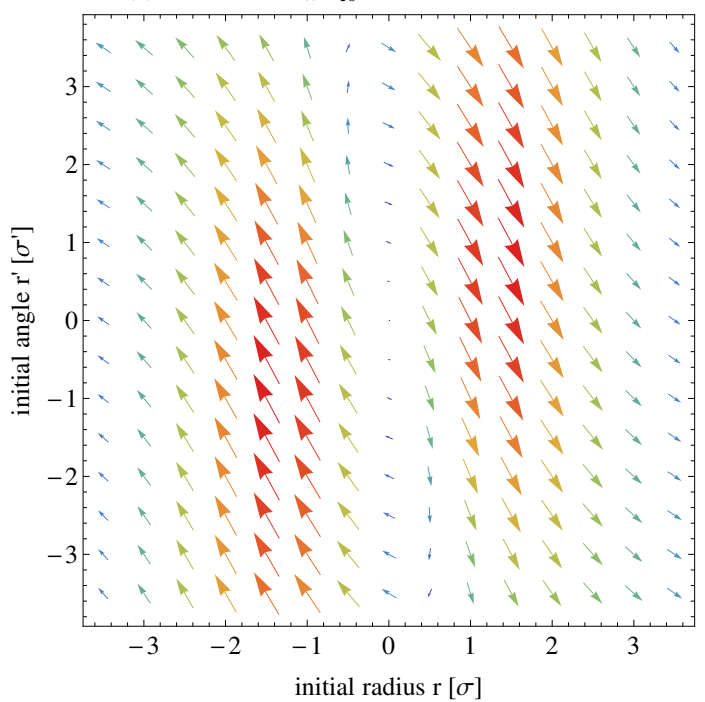

(c) $\mathrm{HOBBC}, \Delta \sigma_{e} / \sigma_{\mathrm{e} 0}=+20 \%$, Max R $=0.0504$

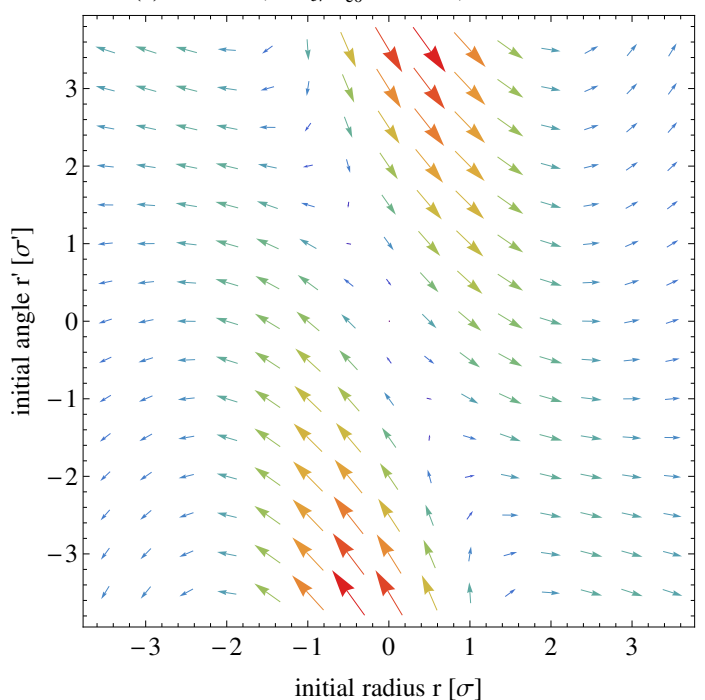

(e) $\mathrm{HOBBC}, \Delta \sigma_{e} / \sigma_{\mathrm{e} 0}=0 \%$, Max $\mathrm{R}=0.0656$

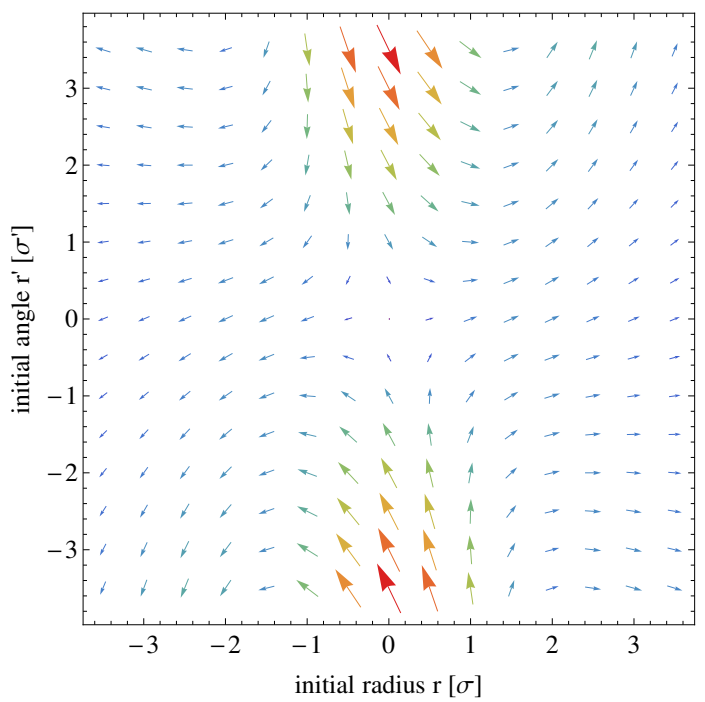

(b) $\mathrm{HOBBC}, \Delta \sigma_{e} / \sigma_{\mathrm{e} 0}=+50 \%$, Max $\mathrm{R}=0.0529$

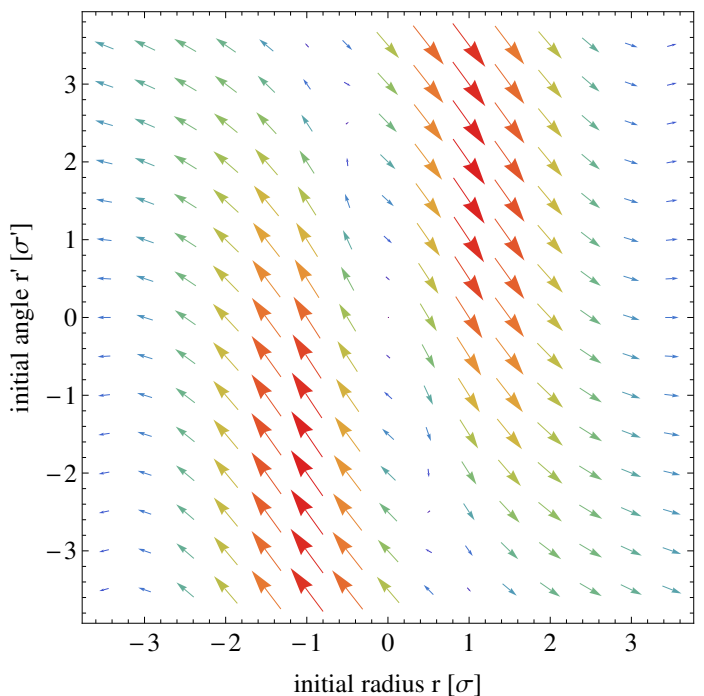

(d) $\mathrm{HOBBC}, \Delta \sigma_{e} / \sigma_{\mathrm{e} 0}=+10 \%$, Max R $=0.0556$

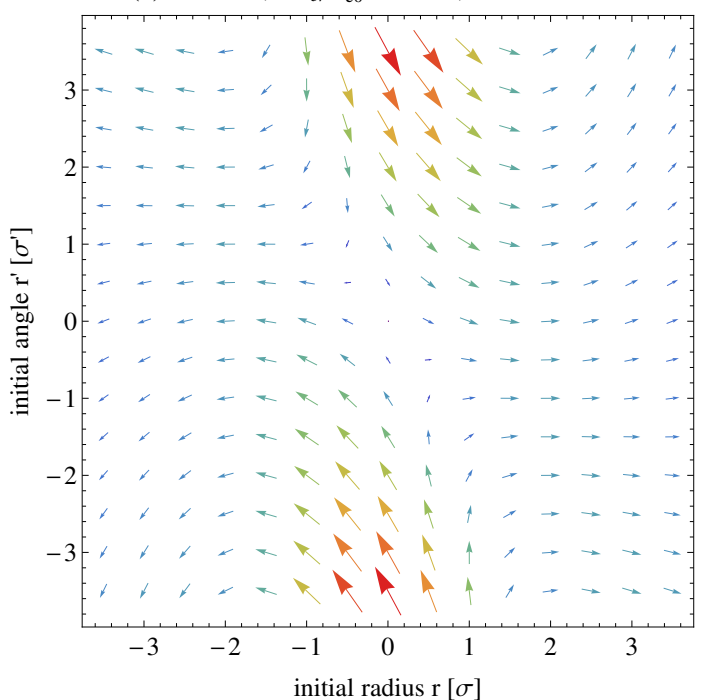

(f) $\mathrm{HOBBC}, \Delta \sigma_{e} / \sigma_{\mathrm{e} 0}=-20 \%$, Max $\mathrm{R}=0.0993$

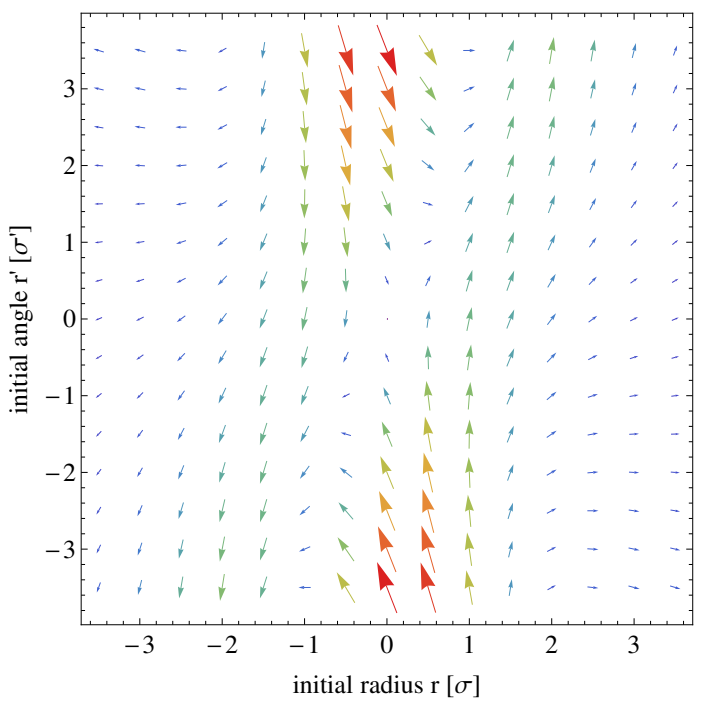

Figure 9: Effect of the electron beam size on $\left(r_{f}, r_{f}^{\prime}\right)$ with HOBBC. $\Delta \sigma_{e} / \sigma_{e 0}=0$ denotes an electron beam size matched to the proton beam size in the center of the electron lens. For all cases, (a) through (f), $\Delta \psi=-10 \mathrm{deg}$. Take note of the $R$ values, $R \equiv 0$ for perfect compensation. 
(a) $\mathrm{HOBBC}, \Delta \mathrm{I}_{e} / I_{\mathrm{e} 0}=+50 \%, \operatorname{Max} \mathrm{R}=0.0731$

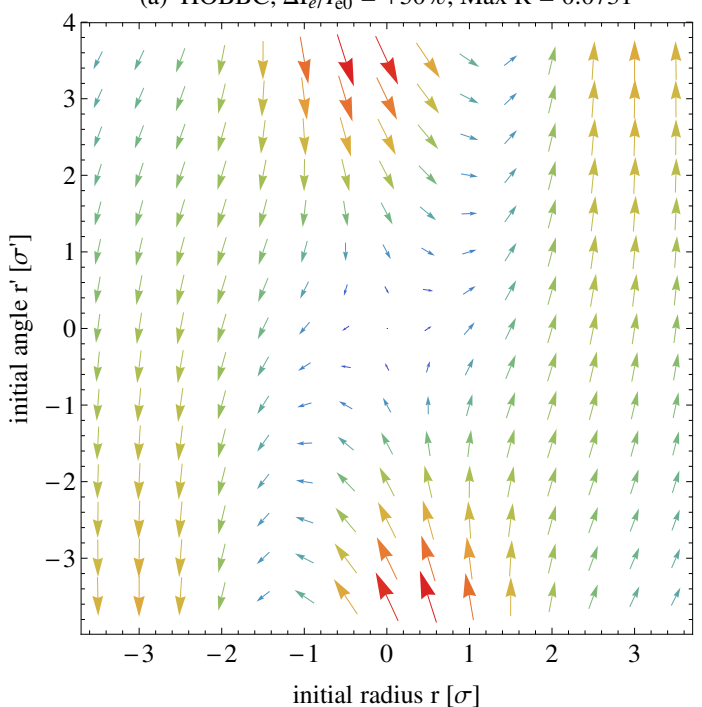

(c) $\mathrm{HOBBC}, \Delta \mathrm{I}_{e} / I_{\mathrm{e} 0}=0 \%, \operatorname{Max} \mathrm{R}=0.0504$

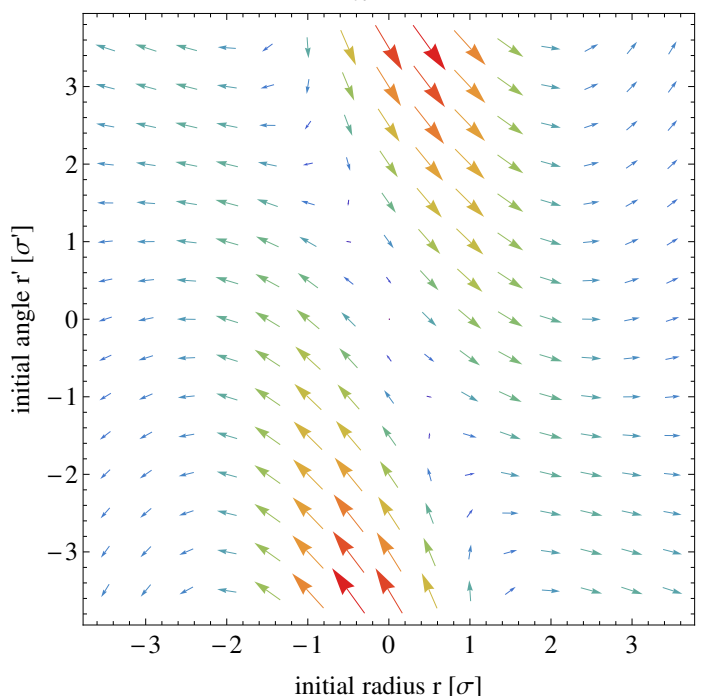

(e) $\mathrm{HOBBC}, \Delta \mathrm{I}_{e} / I_{\mathrm{e} 0}=-20 \%, \operatorname{Max} \mathrm{R}=0.0492$

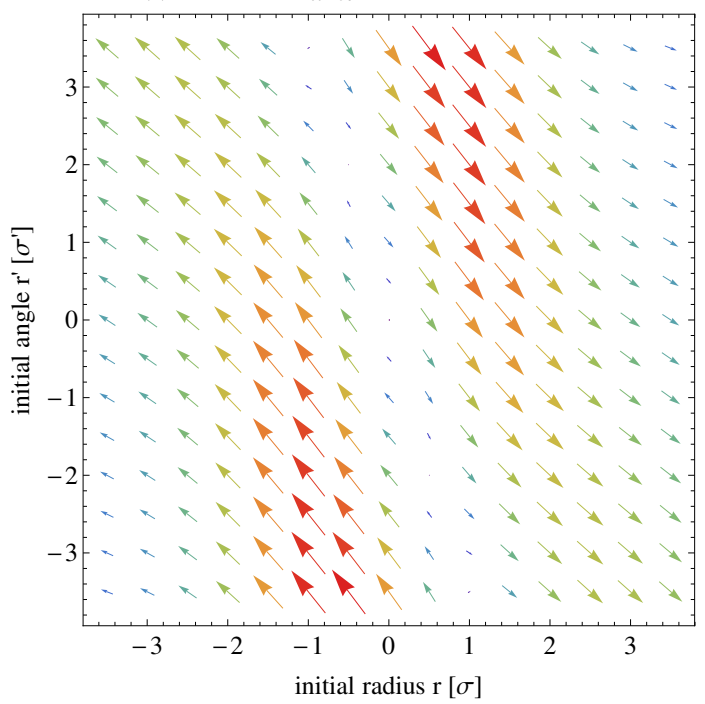

(b) $\mathrm{HOBBC}, \Delta \mathrm{I}_{e} / I_{\mathrm{e} 0}=+10 \%, \operatorname{Max} \mathrm{R}=0.0532$

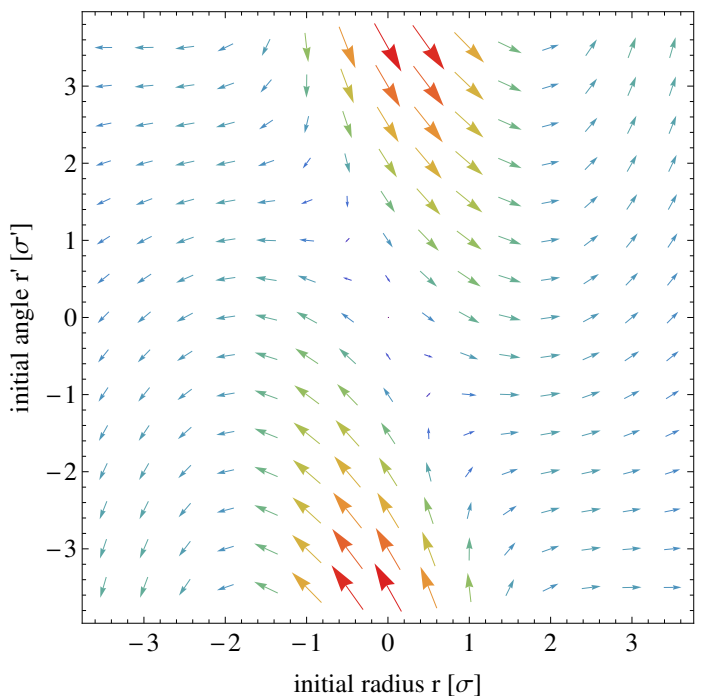

(d) $\mathrm{HOBBC}, \Delta \mathrm{I}_{e} / I_{\mathrm{e} 0}=-5 \%, \operatorname{Max} \mathrm{R}=0.0498$

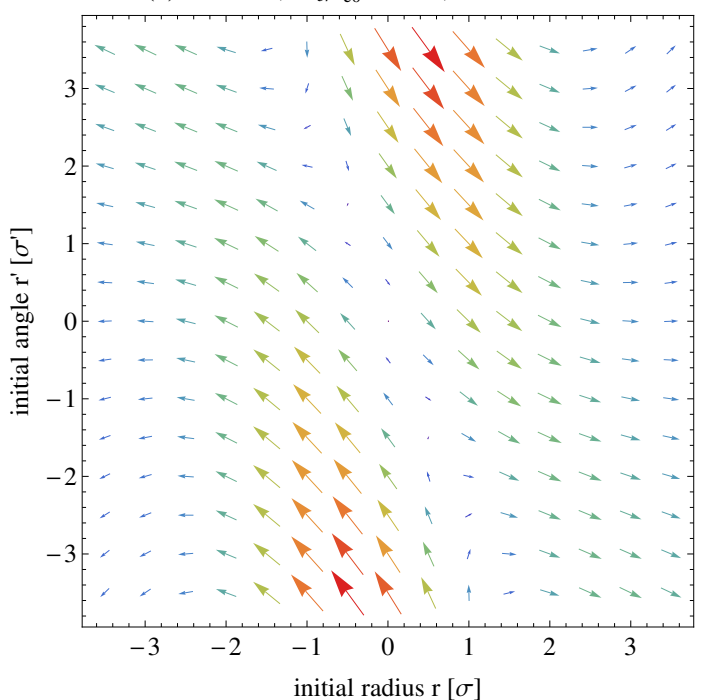

(f) $\mathrm{HOBBC}, \Delta \mathrm{I}_{e} / I_{\mathrm{e} 0}=-50 \%, \operatorname{Max} \mathrm{R}=0.0651$

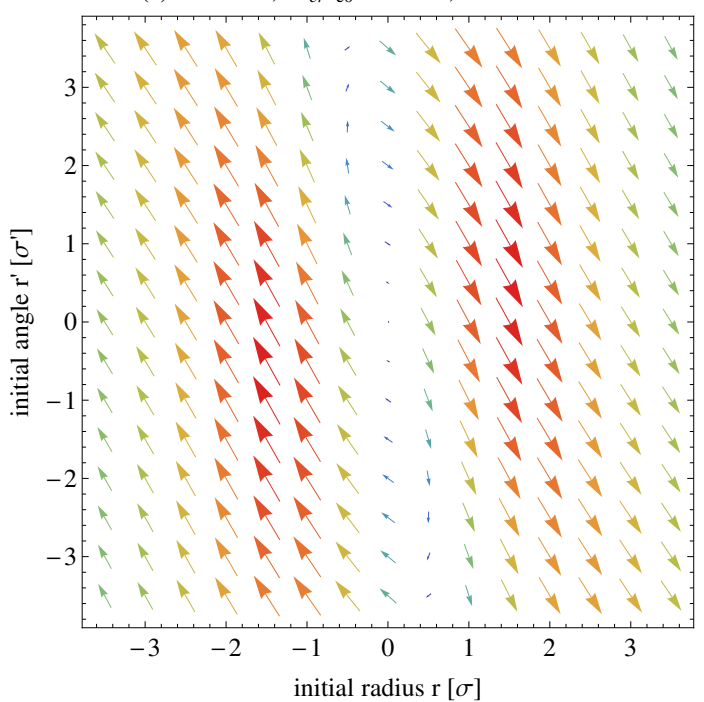

Figure 10: Effect of the electron current on $\left(r_{f}, r_{f}^{\prime}\right)$ with HOBBC. $\Delta I_{e} / I_{e 0}=0$ denotes an electron current matched to the proton bunch intensity $N_{p}$. For all cases, (a) through (f), $\Delta \psi=-10$ deg and $\Delta \sigma_{e} / \sigma_{e 0}=+20 \%$. Take note of the $R$ values, $R \equiv 0$ for perfect compensation. 
(a) no HOBBC, $\delta \mathrm{t}=-3 \sigma_{t}$, Max $\mathrm{R}=0.1120$

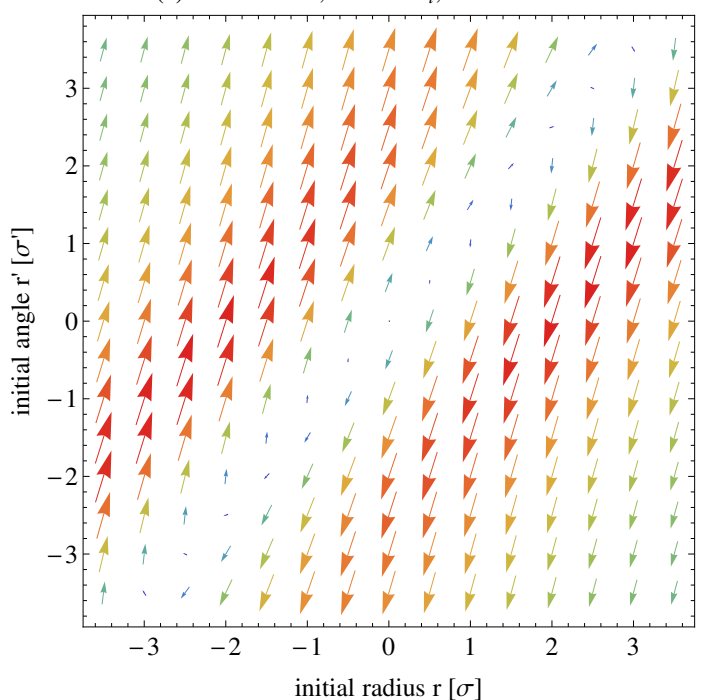

(c) no $\mathrm{HOBBC}, \delta \mathrm{t}=-1 \sigma_{t}$, Max $\mathrm{R}=0.1080$

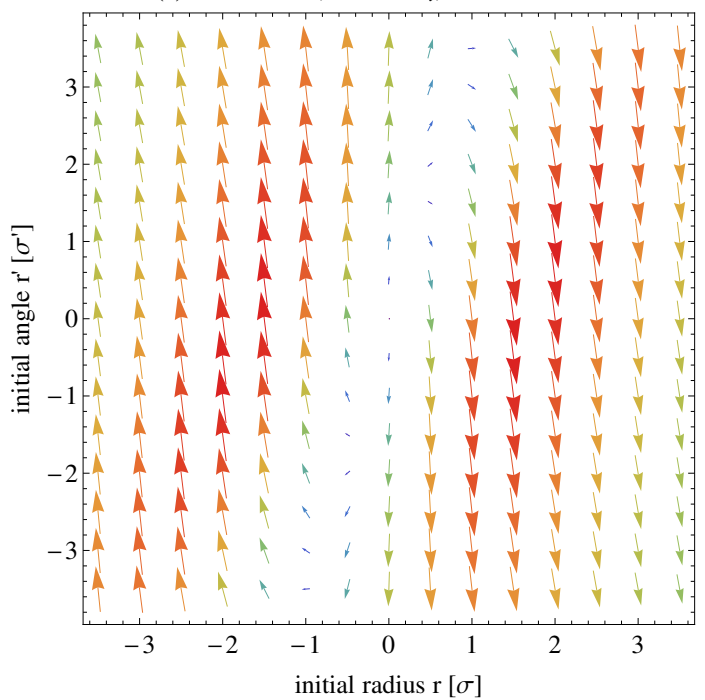

(e) no $\mathrm{HOBBC}, \delta \mathrm{t}=+3 \sigma_{t}, \operatorname{Max} \mathrm{R}=0.1120$

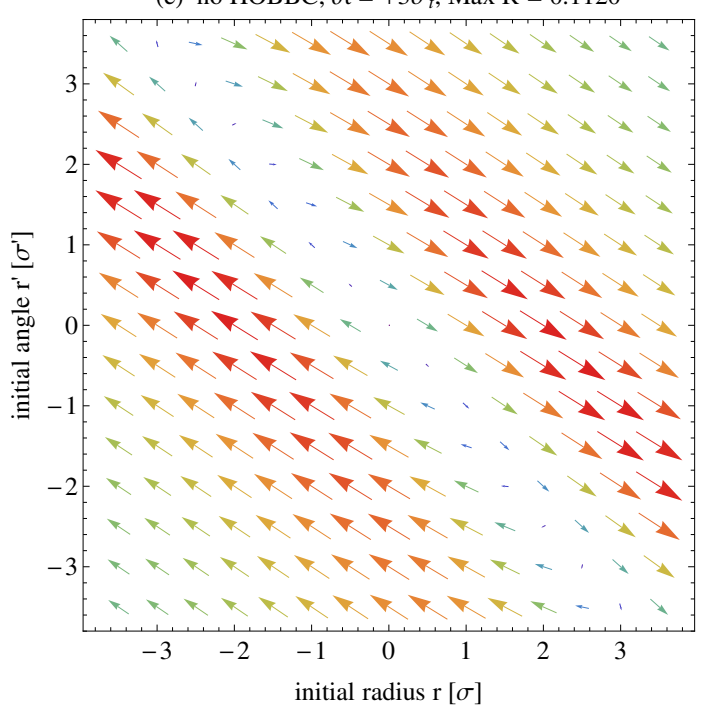

(b) $\mathrm{HOBBC}, \delta \mathrm{t}=-3 \sigma_{t}, \operatorname{Max} \mathrm{R}=0.1350$

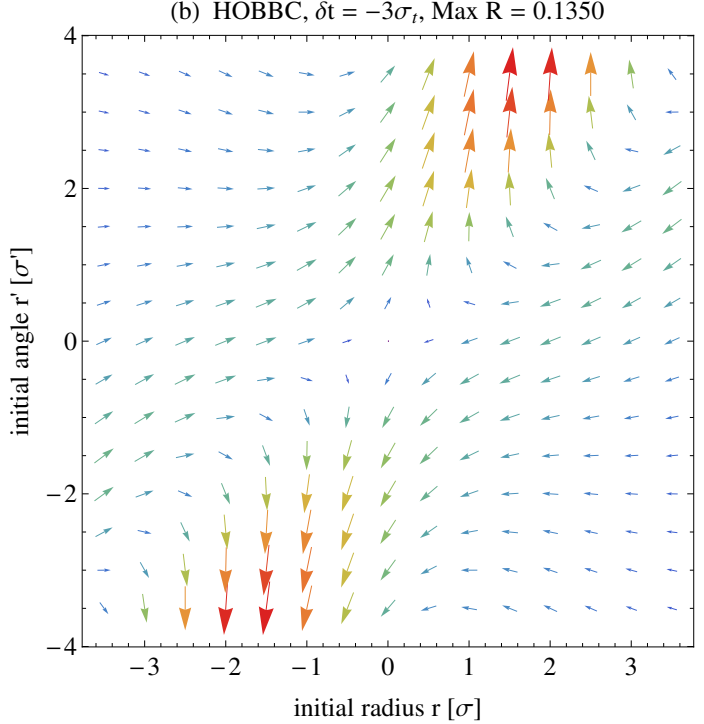

(d) $\mathrm{HOBBC}, \delta \mathrm{t}=-1 \sigma_{t}, \operatorname{Max} \mathrm{R}=0.0315$

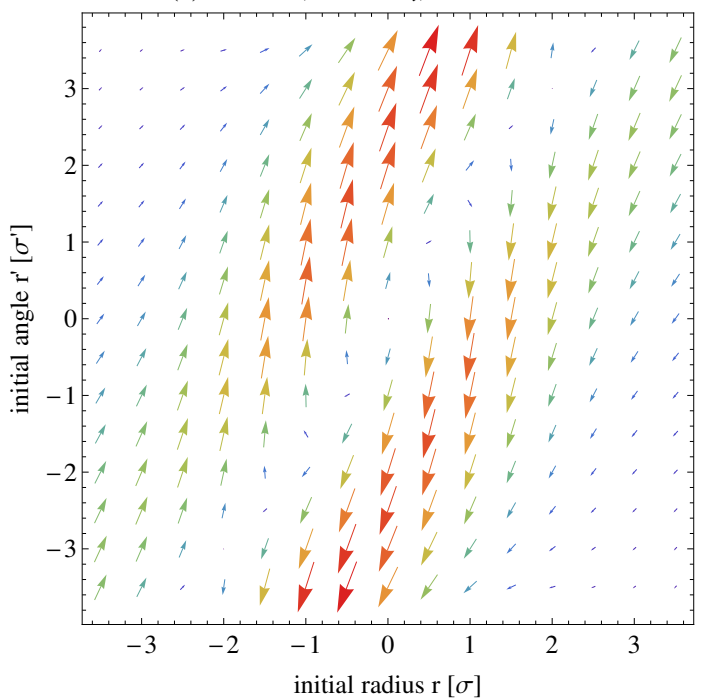

(f) $\mathrm{HOBBC}, \delta \mathrm{t}=+3 \sigma_{t}$, Max $\mathrm{R}=0.1610$

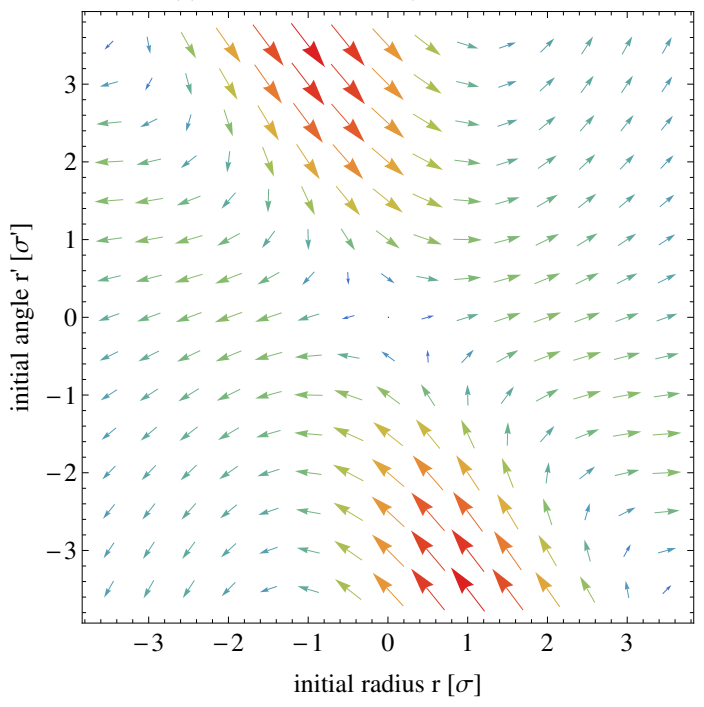

Figure 11: Effect of the particle delay $\delta t$ on the HOBBC. Parts (a), (c), and (e) show $\left(r_{f}, r_{f}^{\prime}\right)$ at IP10 without HOBBC for $\delta t=-3 \sigma_{t},-1 \sigma_{t}$, and $+3 \sigma_{t}$ respectively. Parts (b), (d), and (f) show the $\left(r_{f}, r_{f}^{\prime}\right)$ with HOBBC with $\Delta \psi=-10$ deg, $\Delta \sigma_{e} / \sigma_{e 0}=+20 \%$, and $\Delta I_{e} / I_{e 0}=-5 \%$. Take note of the $R$ values, $R \equiv 0$ for perfect compensation. 